\title{
Clearance of apoptotic $\beta$-cells is reduced in neonatal autoimmune diabetes-prone rats
}

\author{
BA O’Brien ${ }^{1}$, WE Fieldus ${ }^{1}$, CJ Field ${ }^{2}$ and DT Finegood ${ }^{*, 1}$ \\ 1 Diabetes Research Laboratory, School of Kinesiology, Simon Fraser \\ University, Burnaby, British Colmbia, Canada \\ ${ }^{2}$ Agricultural, Food \& Nutritional Science, University of Alberta, Edmonton, \\ Alberta, Canada \\ * Corresponding author: DT Finegood, Diabetes Research Laboratory, School of \\ Kinesiology, Simon Fraser University, Burnaby, British Colombia, V5A 1S6 \\ Canada. Tel: 604-291-3110; Fax: 604-291-3040; E-mail: finegood@sfu.ca
}

Received 7.9.00; revised 24.9.01; accepted 9.10.01

Edited by DR Green

\begin{abstract}
The kinetics of $\beta$-cell death in neonatal diabetes-prone (BBdp) and diabetes-resistant (BBdr) BioBreeding rats was investigated using both direct (histochemical) and indirect (mathematical modelling) techniques. In both BBdp and BBdr rats, the incidence of TUNEL positive $\beta$-cells increased until 10 days of age before declining. The number of apoptotic $\beta$-cells was significantly higher in BBdp as compared to BBdr neonates from birth until 20 days of age $(P<0.05)$. Using a mathematical model applied to the time course of $\beta$-cell mass and replication rate, a wave of net $\beta$-cell loss was detected between 10 and 20 days of age in both strains. In contrast to the observed difference in the incidence of TUNEL positive $\beta$ cells, with the model-based approach we found no difference in the rate of $\beta$-cell apoptosis between BBdp and BBdr rats prior to weaning. As the number of apoptotic cells present in a tissue depends on the rate at which cells die and the rate at which the apoptotic cell debris is cleared, we compared in vitro phagocytosis of apoptotic thymocytes by peritoneal macrophages from 2-week-old BBdp and BBdr rats. Macrophages from BBdp neonates engulfed significantly less apoptotic cells as compared to BBdr neonates $(P<0.0005)$. Taken together, these findings suggest that there is impaired clearance of apoptotic $\beta$-cells in diabetes-prone BB rats during the neonatal period.

Cell Death and Differentiation (2002) 9, 457-464. DOI: 10.1038/ sj/cdd/4400973
\end{abstract}

Keywords: apoptosis; clearance; autoimmunity; diabetes

Abbreviations: BBdp; diabetes-prone BioBreeding; BBdr, diabetes-resistant BioBreeding; NOD; nonobese diabetic; BrdU, 5bromo-2'deoxyuridine; DAB, 3,3'diaminobenzidine tetrahydrochloride; $\mathrm{PI}$, propidium iodide

\section{Introduction}

A multitude of techniques for detecting the hallmark morphological and biochemical features that characterize apoptosis have been developed. ${ }^{1,2}$ Through the application of these methodologies the importance of apoptosis as a mechanism of cell deletion in mature tissue homeostasis, in fetal and neonatal remodeling of organs, and in the pathogenesis of many diseases has been established. ${ }^{3-5}$ Although apoptosis is generally thought to be a noninflammatory event, there is growing evidence that abnormal apoptotic activity occurs in both organ specific and systemic autoimmune disorders. $^{6-8}$

Type 1 diabetes mellitus results from an autoimmunemediated loss of insulin-secreting $\beta$-cells within the pancreatic islets of Langerhans. ${ }^{9}$ It is probable that in individuals with a genetic predisposition, an environmental insult triggers humoral and/or cell-mediated immunities against $\beta$-cells to produce clinical diabetes. $^{10,11}$ The process of $\beta$-cell destruction is chronic in nature, often beginning at a very early age and continuing for several years. During this extensive yet silent preclinical period, inflammatory mononuclear cells infiltrate the pancreatic islets (insulitis) and eliminate the majority of the $\beta$-cell population. Studies using rodent models have shown that $\beta$-cell apoptosis is the primary form of cell loss during the progression to overt type 1 diabetes. ${ }^{12-15}$ We recently hypothesized that neonatal $\beta$ cell apoptosis is also important as a trigger initiating the immune attack. ${ }^{16}$

To study the kinetics of apoptosis, investigators have generally quantified the incidence of apoptotic cells using one of the many available methods such as histochemical detection of a specific marker of the apoptotic process. ${ }^{17}$ Usually, an increase in the appearance of a particular marker for apoptosis (such as TUNEL positivity) is interpreted as an increase in the rate of apoptosis. However, the number of apoptotic cells present in a tissue depends on the rate at which cells die and the rate at which the apoptotic cell debris is cleared. Therefore, defective clearance of apoptotic cells could also be responsible for an increased incidence of apoptotic cells in a tissue. To circumvent this problem, we developed a novel mathematical model-based approach which estimates the rate of cell death based on the rate at which the tissue grows and the rate at which the cells of the tissue replicate. ${ }^{18}$ The rate of cell death calculated in this way is independent of morphological evidence of cell death and thus is independent of the rate at which the apoptotic cells are cleared.

Our hypothesis that neonatal $\beta$-cell apoptosis may be an important trigger for $\beta$-cell directed autoimmunity is based on our prior discovery of a neonatal wave of $\beta$ - 
cell apoptosis in normal developing rats. ${ }^{18-20}$ This neonatal wave of $\beta$-cell death peaks at about 2 weeks of age and is present in both rats and mice. ${ }^{21}$ Studies have pinpointed the initiation of insulitis in diabetes-prone mice at 15 days of age..$^{22}$ The juxtaposition of the neonatal wave and the initiation of autoimmunity led us to the present study in which we sought to determine if the neonatal wave of $\beta$-cell apoptosis is normal in an autoimminue diabetes-prone rat model. The kinetics of $\beta$ cell apoptosis were determined in diabetes-prone (BBdp) and diabetes-resistant (BBdr) BioBreeding rat neonates. We used the TUNEL method to quantify the incidence of apoptotic $\beta$-cells in the pancreas of BBdp and BBdr rats aged 0-25 days. Additionally, we determined the rate of $\beta$-cell death by application of our novel model-based approach. As an independent assessment of apoptotic cell removal in the neonate, we also investigated the ability of peritoneal macrophages from diabetes-prone and diabetes-resistant strains to ingest apoptotic thymocytes in vitro.

\section{Results}

\section{Incidence of apoptotic $\beta$-cells}

The TUNEL method for labelling DNA stand breaks was used in conjunction with insulin staining to quantify the incidence of apoptotic $\beta$-cells in pancreatic sections from BBdr and BBdp rats aged $0-25$ days. Cells with brown nuclei and pink cytoplasm were identified as apoptotic $\beta$ cells (Figure 1). In small apoptotic bodies, the brown colour expanded over the cytoplasm whereas the TUNEL colouration of early apoptotic cells was generally heterogeneous, with reinforcement at the nucleus border. A wave of $\beta$-cell apoptosis was observed during the neonatal period in both strains of BB rat (Figure 2). The \% TUNEL positive $\beta$-cells increased from birth until 10 days of age in both strains. After 10 days of age, values decreased and were significantly lower at 20 and 25 days of age. The number of apoptotic $\beta$-cells in neonatal BBdp rats was significantly greater compared to BBdr neonates between 0 and 20 days of age $(P<0.05)$.

\section{Rate of $\beta$-cell apoptosis}

In order to apply our mathematical model based approach, the rate of change in $\beta$-cell mass and the contribution made by replication were initially determined. $\beta$-Cell mass increased from birth to 10 days of age at a rate of $\sim 0.06 \mathrm{mg} /$ day (Figure $3 \mathrm{~A}$ ). From 10 to 20 days of age, there was no significant change in $\beta$-cell mass in either of the two strains $(P<0.05)$, indicating a plateau in the growth of the $\beta$-cell mass. From 35 to 70 days of age, $\beta$-cell mass was significantly lower in the BBdp animals $(P<0.05)$. BdrU incorporation into $\beta$-cells doubled from $0(1.2 \pm 0.2 \%$ and $1.4 \pm 0.3 \%)$ to 5 days of age $(2.4 \pm 0.3 \%$ and $3.1 \pm 0.2 \%)$ in the BBdp and BBdr rats respectively (Figure $3 \mathrm{~B}$ ). From 5 to 35 days of age, BrdU incorporation generally decreased, although there were abrupt increases at 15 and 30 days of age in the BBdr rats, causing their rate of proliferation to be significantly higher than in the BBdp rats at corresponding ages. Application of the model indicated that at 7.5 days of age, both strains exhibited net cell death which remained until 20 days of age, when the flux of $\beta$-cells turned to net neogenesis of $\sim 7 \% /$ day (Figure 4 ). There was no significant differences in the magnitude of $\beta$-cell death between 10 and 20 days of age with the neonatal wave of cell death averaging $3.4 \pm 1.7 \%$ /day in both strains $(P<0.05)$.
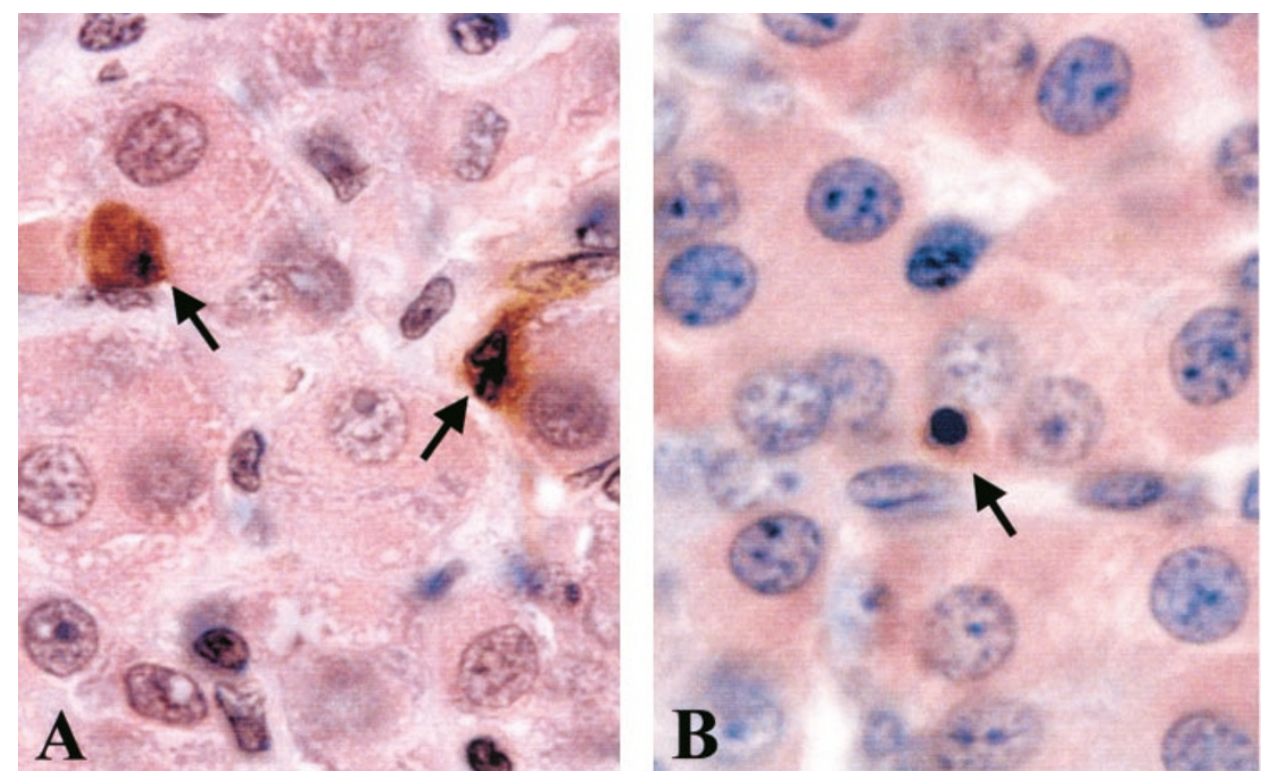

Figure 1 Pictomicrograph showing TUNEL detection of apoptotic $\beta$-cells in Bouins fixed paraffin embedded pancreatic sections from a neonatal BBdp rat at 5 days of age. Cells with brown nuclei and pink cytoplasm were identified as apoptotic $\beta$-cells. Arrows point to early apoptotic $\beta$-cells (A). An apoptotic body with brown TUNEL staining expanded over the cytoplasm is indicated in (B). Original magnification $\times 1000$ 


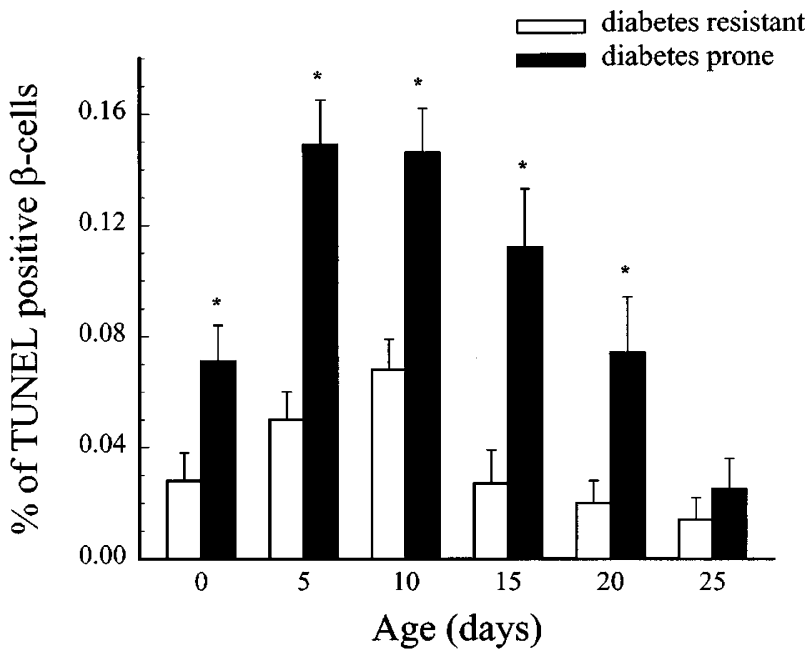

Figure $2 \beta$-cell apoptosis (\% TUNEL positive and insulin positive cells) as a function of age in BBdp and BBdr neonates. Approximately 15000 islet cells for each strain at every time point was observed. ${ }^{*} P<0.05$ between strains
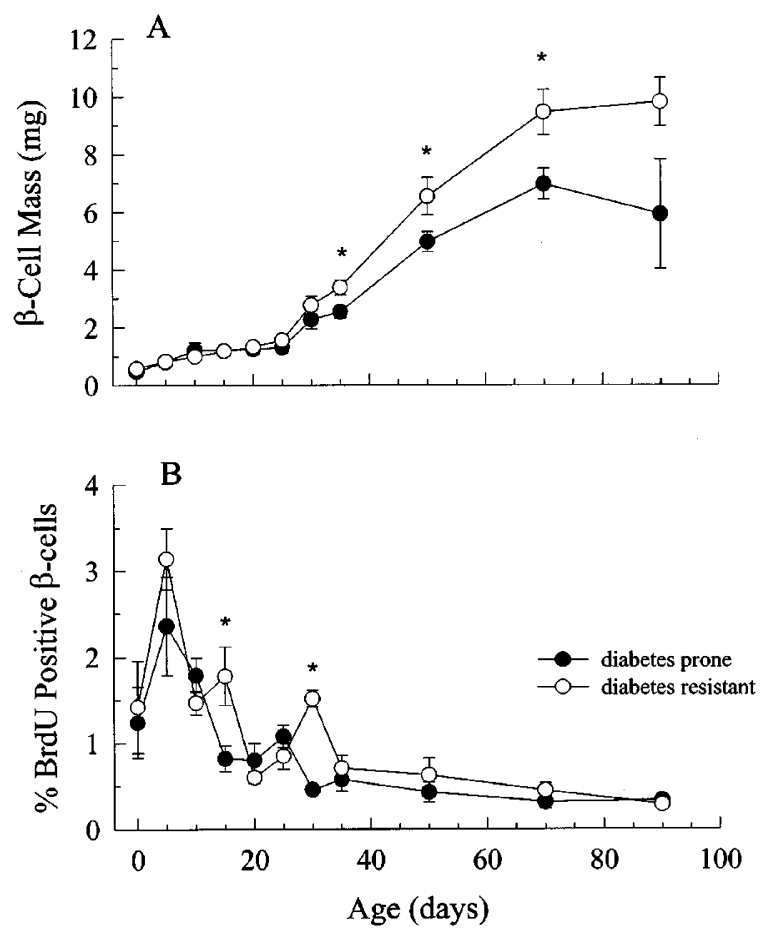

Figure $3 \quad \beta$-cell mass $(\mathbf{A})$ and $B r d U$ labelling index of $\beta$-cells $(\mathbf{B})$ as a function of age in BBdp and BBdr rats. Values presented are means \pm S.E.M. $(n=5$ per age group). ${ }^{*} P<0.05$ between strains

\section{Phagocytosis of apoptotic thymocytes}

Coculture of peritoneal macrophages from 2 week old diabetes-prone and diabetes-resistant BB rats with a fivefold greater number of early apoptotic (Annexin $\mathrm{V}$ positive) thymocytes disclosed a marked deficiency in phagocytosis by macrophages from BBdp rats. Only $2.2 \pm 0.2 \%$ of

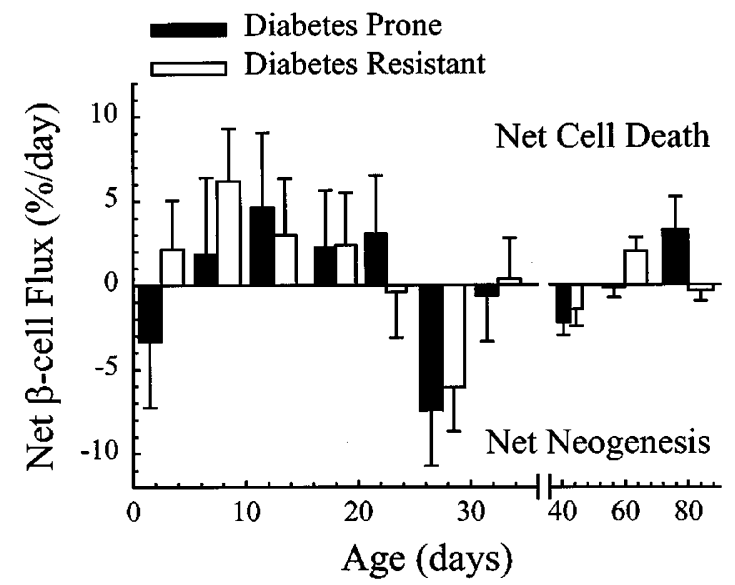

Figure 4 Calculations of net $\beta$-cell neogenesis and net $\beta$-cell death as a function of age in BBdp and BBdr rats as generated by the mathematical model. ${ }^{*} P<0.05$ between strains

peritoneal macrophages from BBdp neonates had ingested at least one apoptotic cell (Figure 5A). In comparison $6.8 \pm 0.9 \%$ of macrophages from age matched BBdr rats were positive for phagocytosis. Phagocytic index (the number of ingested thyocytes per 100 macrophages) also showed a large difference between diabetes-prone and diabetesresistant phenotypes $(2.5 \pm 0.3 \%$ versus $8.2 \pm 1.0 \%$ for $\mathrm{BBdp}$ and BBdr neonates; $P<0.0005$, respectively) (Figure 5B).

\section{Monitoring of diabetes development}

Plasma insulin levels were initially high at birth $(74 \pm 4 \mathrm{pmol} / \mathrm{l})$, but then decreased and remained fairly constant during the neonatal period (Figure 6A). Insulin levels increased from 30 to 70 days of age in both strains. However, during this period, plasma insulin levels were significantly lower in the BBdp as compared to BBdr rats ( $P=0.02$ for 2-way ANOVA from $30-$ 90 days only). Plasma glucose levels were essentially the same between the two strains of BB rats except at 20 days of age (Figure 6B). At 0 days of age, plasma glucose was relatively low at $4.6 \pm 0.1 \mathrm{mmol} / \mathrm{l}$ but then increased to $8.4 \pm 0.2 \mathrm{mmol} / \mathrm{l}$ by 15 days of age. Glucose levels then remained at approximately $9 \mathrm{mmol} / \mathrm{l}$ except in the 90 -day-old BBdp rats when five of 10 animals had developed hyperglycemia (>12 $\mathrm{mmol} / \mathrm{l})$.

\section{Discussion}

Our findings demonstrate, for the first time, that there is a wave of $\beta$-cell apoptosis within the islets of neonatal BBdp and BBdr rats. Both direct (histochemical) and indirect (mathematical modelling) techniques were used to investigate the kinetics of $\beta$-cell death in BBdp and BBdr rats. While our model-based approach suggests that the rate of $\beta$-cell apoptosis is of similar magnitude in both the diabetes-prone and diabetes-resistant strains, direct labelling of DNA strand breaks generated during apoptosis indicates that there is a higher incidence of apoptotic $\beta$-cells in the pancreas of neonatal BBdp rats. The observation that there is an 
diabetes prone

diabetes resistant
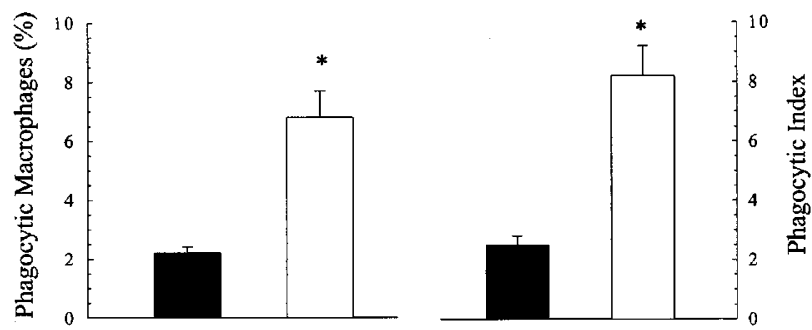

Figure 5 Phagocytosis of apoptotic thymocytes from 2-week-old BBdp and BBdr rats in vitro. Phagocytosis was evaluated by counting 1000 macrophages per animal under oil immersion and data was expressed as phagocytic macrophages (left panel) and phagocytic index (right panel). Values presented are means \pm S.E.M. ( $n=8$ BBdp rats and $n=11 \mathrm{BBdr}$ rats). ${ }^{*} P<0.0005$ between strains
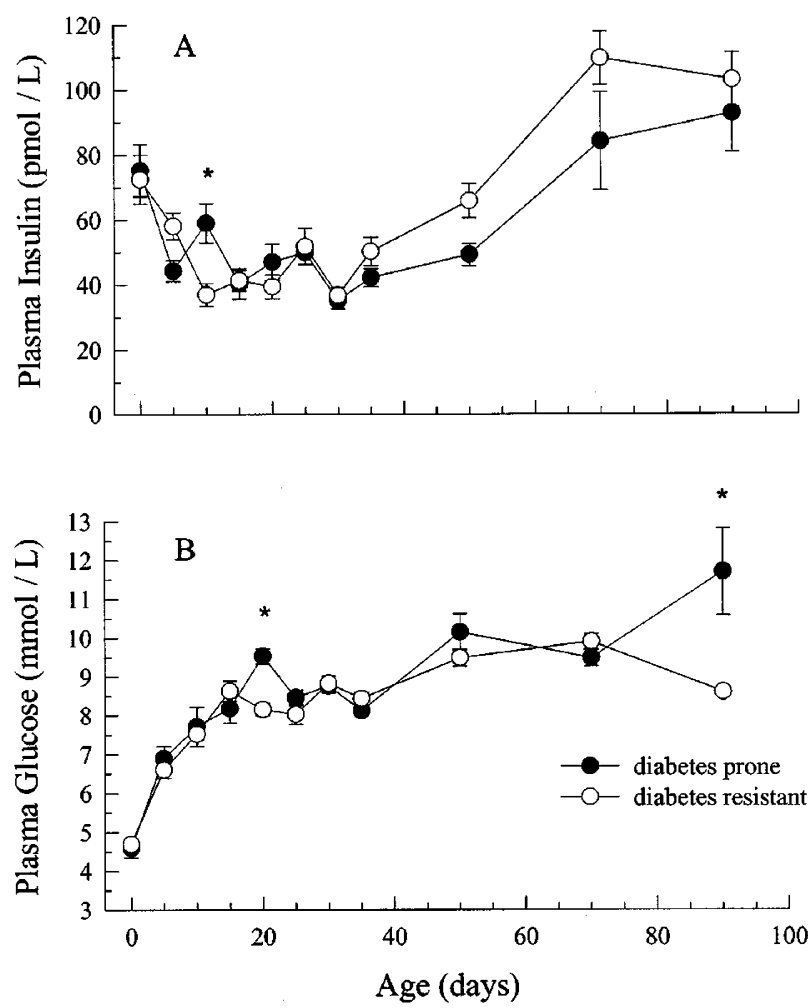

Figure 6 Plasma insulin (A) and glucose (B) levels in male BBdp and BBdr rats 0 to 90 days of age. Data are means \pm S.E.M. ( $n=6-21$ per age group). ${ }^{*} P<0.05$ between strains

increased incidence of apoptotic $\beta$-cells in diabetes-prone animals during the neonatal period is consistent with findings in another autoimmune diabetes-prone model. Using the TUNEL method, we observed an increased number of apoptotic islet cells in neonatal nonobese diabetic (NOD) mice as compared to a diabetes-resistant strain. ${ }^{16}$ However, in this latter study the rate of $\beta$-cell apoptosis was not determined.
An increase in the number of cells displaying a particular marker for apoptosis is often interpreted as an increase in the rate of apoptosis; however, this observation may also be due to a decrease is the rate of clearance by phagocytes. Therefore, the number of TUNEL positive cells observed at a given time point is determined by both the rates of apoptosis and rates of clearance of the apoptotic cells. Our novel model-based approach is independent of the clearance rate of apoptotic cells and hence may be a more accurate reflection of the rate at which cells die. In concordance with this concept, we found that in comparison to macrophages from diabetes-resistant animals, macrophages from diabetes-prone animals displayed a marked deficiency in the ability to ingest apoptotic thymocytes in vitro. The observed difference between the phagocytic ability of macrophages from diabetes-prone and diabetes-resistant strains cannot be explained by differences in the proportion of early apoptotic thymocytes used in phagocytosis assays as the ratio of Annexin $\mathrm{V}$ positive cells to macrophages was always approximately $5: 1$.

In this study only a modest percentage of resident peritoneal macrophages were responsible for all target cell binding (a maximum of $7 \%$ of macrophages from BBdr rats contained at least one apoptotic thymocyte after in vitro phagocytosis assays). It has been reported that even within clonal macrophage populations some macrophages are more competent than others at any given time. ${ }^{23}$ Henry et al. found that in vitro a small subpopulation of macrophages is responsible for the clearance of apoptotic cells. ${ }^{24}$ Subsequent surface marker analysis of this phagocytic subpopulation revealed that they were activated. In our study nonelicited peritoneal macrophages were used and this may have resulted in the low numbers of engulfed apoptotic thymocytes observed. However, we chose to use nonelicited peritoneal macrophages for our experiments as the use of macrophages from other sources either requires prolonged culture or other forms of handling before they can be tested. This carries the intrinsic risk of modulating macrophage function. In the system used in this study the culture time for the macrophages was minimal $(2 \mathrm{~h})$, therefore it is likely that peritoneal macrophages largely maintained their in vivo phenotype. The low phagocytic rates observed for macrophages isolated from BB rats may indicate that a subpopulation is responsible for playing the major phagocytic role.

Macrophages play a major role in the clearance of apoptotic cells and are considered to be the 'professional phagocytes' due to the efficiency with which they engulf and subsequently degrade apoptotic cells. ${ }^{25}$ Macrophages have also been reported to play a pivotal role in the pathogenesis of many autoimmune diseases, ${ }^{26-29}$ including type 1 diabetes where they are among the first immune cells to be activated. ${ }^{30,31}$ In the BB rat model, macrophage infiltration into the islets precedes T- and natural killer (NK)lymphocyte and later B-lymphocyte infiltration. ${ }^{32}$ Further, lymphocytes do not localize to the islets in the absence of prior macrophage invasion. ${ }^{32}$ Functional defects in macrophages isolated from BBdp rats have been reported. Upon stimulation peritoneal macrophages from BBdp rats secrete more tumour necrosis factor-alpha $(\mathrm{TNF}-\alpha)$ than macro- 
phages from BBdr or normal Wistar rats. ${ }^{33}$ TNF- $\alpha$ is an inflammatory cytokine implicated in a number of autoimmune diseases. ${ }^{34}$ Apoptotic cell death is induced by TNF- $\alpha$ in vitro, and has been suggested as one cause of autoimmune pathology. ${ }^{35}$ TNF- $\alpha$ also regulates macrophage activity, which could contribute to autoimmune inflammation. ${ }^{36}$ From the day of birth on, a severe reduction in the thymic macrophage subpopulations has also been observed in BBdp rats as compared to Wistar rats. ${ }^{37}$ Maturational and functional defects in macrophages from diabetes-prone NOD mice have also been identified. ${ }^{38-40}$ Whether these macrophage abnormalities manifest in BBdp rats and NOD mice at a time coincident with the neonatal wave of $\beta$-cell apoptosis to result in decreased clearance of dying $\beta$-cells and initiation of $\beta$-cell directed autoimmunity is currently under investigation. A decrease in the rate of macrophage clearance of apoptotic $\beta$-cells during the neonatal period may be responsible for transforming the physiological wave of $\beta$-cell death into an inflammatory event that initiates $\beta$-cell direct autoimmunity.

Since its original description, ${ }^{41}$ cell death by apoptosis has been assumed to be non-inflammatory for two major reasons. Firstly, the surrounding tissue is protected against the uncontrolled leakage of potentially injurious substances from the dying cell. Secondly, cells undergoing apoptosis in vivo are usually recognized and swiftly ingested by macrophages or neighbouring cells acting as semi-professional phagocytes. Local inflammation and tissue injury are avoided not only because apoptotic cells are prevented from releasing their toxic intracellular components, but also because the macrophage's usual pro-inflammatory secretory response to phagocytosis is not activated ${ }^{42}$ and may be biased towards release of anti-inflammatory cytokines such as transforming growth factor-beta (TFG- $\beta$ ). ${ }^{43}$ Recent studies have emphasized that apoptotic cells are not immunologically inert, but rather have either positive or negative immune effects depending on the antigen presenting cell with which they interact.

Casciola-Rosen et al. ${ }^{44}$ correlated apoptosis and autoimmunity with the observation that antigens for autoantibodies common in systemic lupus erythematosus could be found in distinct clusters in the surface blebs of apoptotic keratinocytes. The selective cleavage of key substrates during apoptosis may reveal immunocryptic epitopes that could potentially induce autoantibody responses in autoimmune diseases. Consistent with this hypothesis, Mevorach et al. ${ }^{45}$ demonstrated that systemic exposure to syngeneic apoptotic thymocytes induces autoantibody production. If macrophages are unable to efficiently clear apoptotic debris then dendritic cells may be recruited to perform phagocytic functions with subsequent T-lymphocyte activation. Albert et al. ${ }^{46}$ showed that immature dendritic cells can cross-present antigens to cytotoxic Tlymphocytes after phagocytosis of apoptotic cells. Collectively, these studies indicate that apoptotic cells can display autoreactive antigen, preferentially activate dendritic cells that are capable of priming T-lymphocytes, and induce the formation of autoantibodies.

That the neonatal wave of $\beta$-cell death might be responsible for the uncovering of novel antigen is also supported by the studies of Hoglund et al. ${ }^{22}$ These authors demonstrated that a change takes place in the pancreas after 10 days of age, which allows for antigen recognition and T-lymphocyte proliferation. Naïve $\beta$-cell specific $\mathrm{T}$ lymphocytes from either 10-day-old or adult transgenic mice (BDC2.5) proliferate when given to adult NOD mice. In contrast, naïve T-lymphocytes from adult transgenic mice do not proliferate when transferred into a 10-day-old recipient. This suggests that the T-lymphocytes of 10-dayold mice are competent, however there is insufficient antigen being presented for recognition at 10 days of age. Additional studies demonstrated that T-lymphocytes begin to proliferate at approximately 15 days of age suggesting that some phenomenon occurs and reveals antigen between 10 and 15 days of age, a time coincident with the neonatal wave.

The collective findings of the present study strongly suggest that there is impaired clearance of apoptotic $\beta$-cells in diabetes-prone BB rats during the neonatal period. The observed defective engulfment of apoptotic cell material in type 1 diabetes could have a major impact on immune pathogenic mechanisms. Disruption of the dynamic equilibrium between the production and clearance of apoptotic cells will potentially expose the immune system to harmful autoantigens generated during the apoptotic process. A more complete understanding of abnormalities in the execution of the apoptotic program and of the clearance defects inherent to antigen presenting cells may provide potential mechanisms for modifying the pathogenesis of autoimmune diseases.

\section{Material and Methods}

\section{Animals}

All procedures were in accordance with the standards set by the Canadian Council on Animal Care and were approved by animal care committees at the University of Alberta and Simon Fraser University. BioBreeding rats were obtained from the University of Alberta, Department of Agricultural, Food and Nutritional Science breeding colony. Original breeding pairs were obtained from Health Canada (Animal Resources Division, Health Protection Branch, Ottawa, ONT, Canada). The incidence of diabetes in this colony is approximately $80-85 \%$ in both male and female rats with a mean diabetes age of onset at 96 days. Diabetes was diagnosed by regular urine tests for glucosuria. Rats were given free access to standard laboratory chow (NIH-07 meal diet; Zeigler Brothers, Gardners, PA, USA) and water ad libitum. Animals were bred specifically for this study and litters were culled to 8-10 pups to minimize differences in nutrition prior to weaning. Male and female pups were removed from the dam and weaned onto chow at 21 days of age. All animals from a given litter were killed on the same day.

\section{Experimental design}

Animals of $0,5,10,15,20,25,30,35,50,70$ and 90 days were used. The thymidine analogue 5-bromo- 2 'deoxyuridine (BrdU, $100 \mathrm{mg} / \mathrm{kg}$, Sigma Diagnostics, St. Louis, MO, USA) was injected intraperitoneally $6 \mathrm{~h}$ before removal of the pancreas. For the terminal procedure, non- 
fasted rats were deeply anaesthetized with halothane. Blood was sampled by cardiac puncture, the pancreas was removed and cutting the diaphragm killed the animal.

\section{Plasma glucose and insulin determination}

Blood samples were stored in heparinized tubes on ice until centrifugation. Plasma samples were stored at $-20^{\circ} \mathrm{C}$ until they were analyzed for glucose using a glucose oxidase method (Trinder Kit, Sigma Diagnostics, St Louis, MO, USA) and insulin was measured by radioimmunoassay using rat insulin standards (Linco, St Louis, MO, USA). All samples were assayed in duplicate. The coefficient of variation (CV) for duplicate plasma glucose determinations was $3.5 \pm 4.5 \%$ (mean \pm S.D.). The within assay CV for plasma insulin determinations was $3.6 \pm 4.2 \%$ and the between assay CV was $6.3 \pm 1.8 \%$. A total of 272 animals from 112 litters were used for determination of plasma glucose and 269 animals from 97 litters were used for determination of plasma insulin levels.

\section{Histology}

For each strain at each age, tissue samples from five animals were randomly selected. In total, 110 animals were used from a minimum of four litters at each age and strain. The pancreas was removed from the animal, quickly cleared of adipose tissue, and then weighed and placed in Bouins fixative overnight. Pancreas from older animals were divided into two or three sections before being weighed, to facilitate the absorption of fixative. After fixation, the tissue samples were washed in cold water and then stored in $10 \%(\mathrm{w} / \mathrm{v})$ buffered formalin until embedding in paraffin. Six serial sections of pancreas $(4 \mu \mathrm{m}$ each) were cut. In animals aged 0 to 25 days, a minimum of four sets of serial sections, separated by at least $40 \mu \mathrm{m}$, were prepared to allow for assessment of similar cross sectional areas across the range of age groups.

\section{Identification and quantification of $\beta$-cell apoptosis}

The terminal deoxynucleotidyl transferase (TdT)-mediated deoxyuridine triphosphate (dUTP)-nick end labeling (TUNEL) method for labeling DNA strand breaks was used to quantify the number of apoptotic $\beta$-cells in section of pancreas from BBdp and BBdr rats aged $0-25$ days ( $n=5$ animals at each age and strain). Slides were placed in a glass container $200 \mathrm{ml}$ of $0.01 \mathrm{M}$ citrate buffer $(\mathrm{pH} 6)$ and exposed to microwave irradiation for $5 \mathrm{~min}$, resulting in elevation of the temperature to $80^{\circ} \mathrm{C}$. Immediately after irradiation, the container was filled with an extra $80 \mathrm{ml}$ of distilled water to rapidly cool the antigen retrieval solution. The ApopTag kit (Intergen, Purchase, NY, USA) which utilizes digoxygenin (DIG)-dUTP and a peroxidase-labelled antiDIG antibody was then used to identify the apoptotic cells. Visualization of positive cells was achieved with DAB as the substrate. To provide a negative control of the TUNEL, TdT was omitted from the labeling mix. The $\beta$-cell origin of the apoptosis was verified by double labelling with antibodies to insulin and visualizing with the chromogen New Fuschin as described below. Cells with brown nuclei (TUNEL positive) and pink cytoplasm (insulin positive) were identified as apoptotic $\beta$-cells.

Apoptosis was quantified in all $\beta$-cells present in stained sections from each animal, each section being separated from the next by at least $40 \mu \mathrm{m}$. A total of $3012 \pm 37$ (mean \pm S.E.M.) $\beta$-cells were counted for each animal at each time point. A single individual (BAO) carried out analysis in a blinded fashion. The number of apoptotic $\beta$-cells present was expressed as a percentage of the total number of $\beta$-cells observed. Results were expressed as mean values of animals from each strain at each time point \pm standard error of the mean (S.E.M.) between individuals.

\section{$\beta$-cell mass}

$\beta$-cell mass was determined on sections of pancreas immunostained with anti-insulin antibody only. Sections were randomized and relabelled to ensure blinded counting. Samples were analyzed with a light microscope (final magnification $=172 \times$ ) and image analysis software (Northern Eclipse, Empix Imaging, Mississauga, ONT, Canada). Tissue sections were stepped through systematically; an average of $82 \pm 25$ (mean \pm S.D.) fields were quantified per animal. The image analysis software was used to trace and select by thresholding immunostained $\beta$-cell area. Non pancreas areas of fat and connective tissue were determined to allow for correction of the pancreas weights. Corresponding areas from each field were summed and compared to the total tissue area in order to calculate the relative $\beta$-cell area. This per cent was then multiplied by the total wet weight of the tissue to give an estimate of $\beta$-cell mass.

\section{$\beta$-cell replication}

To determine the $\beta$-cell replication rate, sections of pancreas were double stained for BrdU and insulin. Sections were incubated for $1 \mathrm{~h}$ at $4^{\circ} \mathrm{C}$ with a mouse monoclonal anti-BrdU antibody (1: 100, Amersham, Arlington Heights, IL, USA) in a solution containing nuclease for DNA denaturation. Samples were then washed with phosphate buffered saline (PBS, $\mathrm{pH}=7.4$ ), incubated with a biotinylated anti-mouse antibody $(1: 500$, Vector, Burlington, ONT, Canada) followed by an avidin/peroxidase complex $(1: 2000$, Vector, Burlington, ONT, Canada) and finally stained with 3,3'diaminobenzidine tetrahydrochloride (DAB) (Sigma, Oakville, ONT, Canada). The sections were then washed in PBS again and subsequently stained for insulin using guinea pig anti-porcine insulin antibody $(1: 2000$, Dako, Mississauga, ONT, Canada) for $24 \mathrm{~h}$ at $4^{\circ} \mathrm{C}$. The sections were then washed with PBS, incubated with a biotinylated anti-guinea pig $\lg \mathrm{G}$ as secondary antibody (1:500, Vector, Burlington, ONT, Canada), incubated with an avidin/alkaline phosphatase complex $(1: 2000$, Vector, Burlington, ONT, Canada) and stained with the chromogen New Fuschin (Dako, Mississauga, ONT, Canada).

Samples were randomized and re-labelled to ensure a blinded counting procedure. A single individual (WEF) counted all samples. Using a light microscope under $100 \times$-oil immersion objective, all BrdU positive and negative $\beta$-cell nuclei were counted $(3539 \pm 1773$ (mean \pm S.D.) nuclei per animal).

\section{Net neogenesis and net cell death}

Net neogenesis and net cell dath were calculated using our previously described mathematical model for $\beta$-cell turnover.

$$
\text { Neogenesis Cell Death }=\mathrm{d}(\beta \quad \text { cell \# }) / \mathrm{dt} \text { Replication }
$$

$\beta$-Cell size was assumed to be constant and the BrdU labelling index (LI) was converted to a replication rate (Replication $=\left(\mathrm{LI}^{*} 24 \mathrm{~h}\right) / 6 \mathrm{~h}$ ). Since $\beta$-cell size and density were assumed to be relatively constant, the rate of change in $\beta$-cell number $(\mathrm{d}(\beta$-cell $\#) / \mathrm{dt})$ is equal to the rate of change of $\beta$-cell mass. When the replication rate is greater than the rate of change of the $\beta$-cell mass, the model indicates that net cell loss must occur. Conversely, when the $\beta$-cell mass increases at a faster rate than can be accounted for by replication, net neogenesis is indicated. 


\section{Phagocytosis assays}

Nonelicited peritoneal macrophages were obtained from 2-week-old male BBdp ( $n=8$; collected from two litters) and BBdr ( $n=11$; collected from three litters) neonates by closed peritoneal lavage with ice-cold RPMI media (RPMI 1640 medium containing $100 \mathrm{U} / \mathrm{ml}$ penicillin, $100 \mathrm{U} / \mathrm{ml}$ streptomycin, $10 \% \mathrm{v} / \mathrm{v}$ heat inactivated foetal caf serum (FCS) (Gibco Life Technologies, NY, USA). Cells were washed, resuspended in fresh medium, and seeded at $2 \times 10^{5}$ cells per well into 8-well chamber slides (Nalge Nunc, IL, USA). Peritoneal cells were incubated for $2 \mathrm{~h}$ at $37^{\circ} \mathrm{C}, 5 \% \mathrm{CO}_{2}$ in a humidified incubator to allow macrophages to adhere and spread. Nonadherent cells were removed by washing three times with warmed RPMI medium, after which $500 \mu \mathrm{l}$ of medium was immediately added to each well. To ensure that equal numbers of adherent macrophages among strains remained after washing, nonadherent cells from washes of single cells were routinely counted and showed no differences among strains.

Autologous thymocytes were used for phagocytosis assays. Rat thymuses were harvested and minced to yield a single cell suspension. The thymocytes were irradiated with a UV lamp for $20 \mathrm{~min}$ and then cultured in RPMI/serum-free medium for $2 \mathrm{~h}$ at $37^{\circ} \mathrm{C} /$ $5 \% \mathrm{CO}_{2}$. Cell death was confirmed using FITC-labelled Annexin V (Molecular Probes, OR, USA) and propodium iodide (PI) (Molecular Probes, OR, USA) staining and observation under a fluorescent microscope. Two hours after irradiation, a large proportion of the thymocyte population had externalized PS on the outer leaflet of their cell membrane ( $42 \pm 6 \%$ Annexin $V$ positive; $12 \pm 3 \% \mathrm{PI}$ positive).

Thymocytes were cocultured with macrophages, at a ratio of $5: 1$, Annexin $\mathrm{V}$ positive thymocytes : macrophages in RPMI supplemented with $20 \%$ heat-inactivated $\mathrm{FCS}$ for $60 \mathrm{~min}$ at $37^{\circ} \mathrm{C}$ in $5 \% \mathrm{CO}_{2}$. After phagocytosis, the wells were washed three times with ice cold PBS to remove thymocytes that had not been phagocytosed and then fixed in neutral buffered formalin. Cells were stained with hematoxylin and eosin $Y(H$ \& E) and observed by light microscopy. Phagocytosis was assessed by counting 1000 macrophages per well at $1000 \times$ magnification under oil immersion. Only thymocytes clearly within the perimeter of the macrophage were counted. Results were expressed as percentage of macrophages containing at least one ingested thymocyte (phagocytic macrophages) and as phagocytic index (total number of apoptotic bodies per 100 macrophages).

\section{Statistical analysis}

The results of plasma insulin, plasma glucose, $\beta$-cell mass, $\beta$-cell replication and insulitis were analyzed using a 2-way analysis of variance (ANOVA) by age and strain. The error associated with the flux calculation (net neogenesis and net cell death) was determined by a bootstrapping procedure. Individual estimates of $\beta$-cell mass in the five animals from each of two sequential points in time were used to determine all possible combinations of points. The slope of these segments was used to calculate the mean and variance of the rate of change in the mass. These values apply most appropriately to the point in time midway between the two sample times. As such the net flux calculation was based on the rate of change and the mean rate of replication between each two sequential points in time. Differences in net flux between strains were determined by an unpaired $t$-test. A value of $P<0.05$ was considered significant. Results are expressed as mean \pm standard error of the mean (S.E.M.) unless otherwise noted.

\section{Acknowledgements}

The authors are grateful to Dan Tzur, Narinder Dhatt and Lily Huang for their technical assistance. This study was supported by funds from the
Canadian Institutes of Health Research/Juvenile Diabetes Research Foundation International. BA O'Brien is supported by a Juvenile Diabetes Research Foundation International Postdoctoral Fellowship. BA O'Brien and DT Finegood are members of the Beta Cell Apoptosis Network (BetaCAN). A portion of the data was published in preliminary form as part of a 'Perspectives' article in Diabetes. ${ }^{16}$

\section{References}

1. Harmon BV, Winterford CM, O'Brien BA and Allan DJ (1997) Morphological criteria for identifying apoptosis. In Cell Biology. A Laboratory Handbook. Volume 1. Celis JE, ed. (New York: Academic Press)

2. Saraste A and Pulkki K (2000) Morphologic and biochemical hallmarks of apoptosis. Cardiovasc. Res. 45: 528-537

3. James TN (1994)Normal and abnormal consequences of apoptosis in the human heart. From postnatal morphogenesis to paroxysmal arrhythmias. Circulation 90: $556-573$

4. Hardy K (1999) Apoptosis in the human embryo. Rev. Reprod. 4: 125-134

5. Kerr JF, Winterford CM and Harmon BV (1994) Apoptosis: Its significance in cancer and cancer therapy. Cancer 73: 2013-2026

6. Suvannavejh GC, Dal Canto MC, Matis LA and Miller SD (2000) Fas-mediated apoptosis in clinical remissions of relapsing experimental autoimmune encephalomyelitis. J. Clin. Invest. 105: 223-231

7. Pablos JL, Santiago B, Galindo M, Carreira PE, Ballestin C and Gomez-Reino JJ (1999) Keratinocyte apoptosis and p53 expression in cutaneous lupus and dermatomyositis. J. Pathol. 188: $63-68$

8. Phelps E, Wu P, Bretz J and Baker Jr JR (2000) Thyroid cell apoptosis. A new understanding of thyroid autoimmunity. Endocrinol. Metab. Clin. North Am. 29: $375-388$

9. Gepts W and Lecompte PM (1980) The pancreatic islets in diabetes. Am. J. Med. 70: $105-115$

10. Eisenbarth GS (1986) Type I diabetes mellitus: A chronic autoimmune disease N. Engl. J. Med. 21: 1360-1368

11. Nerup J, Mandrup-Poulsen T, Pociot F, Karlsen AE, Andersen HU, Christensen UB, Sparre T, Johannesen J and Kristensen OP (1997) On the pathogenesis of insulin-dependent diabetes mellitus in man: A paradigm in transition. In: Contributions of Physiology to the Understanding of Diabetes. Zahnd GR and Wollhein CB, eds. (London: Springer-Verlag) pp. 148-159

12. O'Brien BA, Harmon BV, Cameron DP and Allan DJ (1997) Apoptosis is the mode of beta cell death responsible for the development of IDDM in the nonobese diabetic (NOD) mouse. Diabetes 46: 750-757

13. O'Brien BA, Harmon BV, Cameron DP and Allan DJ (1996) Beta cell apoptosis is responsible for the development of IDDM in the multiple low-dose streptozotocin model. J. Path. 178: 176-181

14. Chervonsky AV, Wang Y, Wong FS, Vistinin I, Flavell RA, Janeway Jr CA and Matis LA (1997) The role of Fas in autoimmune diabetes. Cell 89: 17-24

15. O'Brien BA, Harmon BV, Cameron DP and Allan DJ (2000) Nicotinamide prevents the development of diabetes in the cyclophosphamide-induced NOD mouse model by reducing beta-cell apoptosis. J. Pathol. 191: 86-92

16. Trudeau JD, Dutz JP, Arany E, Hill DJ, Fieldus WE and Finegood DT (2000) Neonatal $\beta$-cell apoptosis: A trigger for autoimmune diabetes? Diabetes 49:1 - 7

17. Huppertz B, Frank HG and Kaufmann P (1999) The apoptosis cascademorphological and immunohistochemical methods for its visualization. Anat. Embryol. 200: 1 -18

18. FinegoodDT, Scaglia L and Bonner-Weir S(1995) Dynamics of $\beta$-cell mass in the growing rat pancreas. Estimation with a simple mathematical model. Diabetes 44: $249-256$

19. Scaglia L, Cahill J, Finegood DT and Bonner-Weir S (1997) Apoptosis participates in the remodeling of the endocrine pancreas in the neonatal rat. Endrocrinology 138: 1736-1741

20. PetrikJ, Arany E, McDonald J and Hill DJ (1998) Apoptosis in the pancreatic islet cells of the neonatal rat is associated with a reduced expression of insulin-like growth factor II that may act as a survival factor. Endocrinology 139: 2994-3004

21. Hill DJ, Strutt B, Arany E, Zaina S, Coukell S and Graham CF (2000) Increased and persistent circulating levels of insulin-like growth factor II in neonatal transgenic mice suppresses developmental apoptosis in the pancreatic islets. Endocrinology 141: 1151-1157 
22. Hoglund P, Mintern J, Waltzinger C, Heath W, Benoist C and Mathis D (1999) Initiation of autoimmune diabetes by developmentally regulated presentation of islet cell antigens in the pancreatic lymph nodes. J. Exp. Med. 189: 331-339

23. Cocco RE and Ucker DS (2001) Distinct modes of macrophage recognition for apoptotic and necrotic cells are not specified exclusively by phosphatidylserine exposure. Mol. Biol. Cell 12: 919-930

24. Henry F, Bretaudeau L, Barbieux I, Meflah K and Gregoire M (1998) Induction of antigen presentation by macrophages after phagocytosis of tumour apoptotic cells. Res. Immunol. 149: 673-679

25. Wyllie AH, Kerr JFR and Currie AR (1980) Cell death: The significance of apoptosis. Int. Rev. Cytol. 68: 251-306

26. Kitamura T, Tanaka N, Watanabe J, Uchida K, Kanegasaki S, Yamada Y and Nakata K (1999) Idiopathic pulmonary alveolar proteinosis as an autoimmune disease with neutralizing antibody against granulocyte/macrophage colonystimulating factor. J. Exp. Med. 190: 875-880

27. Mustafa W, Zhu J, Deng G, Diab A, Link H, Frithiof L and Klinge B (1998) Augmented levels of macrophage and Th1 cell-related cytokine mRNA in submandibularglands of MRL/lpr mice with autoimmune sialoadenitis. Clin. Exp. Immunol. 112: 389-396

28. Martiney JA, Rajan AJ, Charles PC, Cerami A, Ulrich PC, Macphail S, Tracey KJ and Brosnan CF (1998) Prevention and treatment of experimental autoimmune encephalomyelitis by $\mathrm{CNI}-1493$; a macrophage-deactivating agent. J. Immunol. 160: $5588-5595$

29. Kiefer R, Kieseier BC, Bruck W, Hartung HP and Toyka KV (1998) Macrophage differentiation antigens in acute and chronic autoimmune polyneuropathies. Brain 121: $469-479$

30. Kolb-Bachofen V, Epstein S, Kiesel U and Kolb H (1988) Low dose streptozotocin-induced diabetes in mice. Electron microscopy reveals single cell insulitis before diabetes onset. Diabetes 37: 21-27

31. Lee KU, Amano K and Yoon JW (1988) Evidence for initial involvement of macrophage in development of insulitis in NOD mice. Diabetes 37: 989-991

32. Hanenberg H, Kolb-Bachofen V, Kantwerk-Funke G and Kolb H (1989) Macrophage infiltration precedes and is a prerequisite for lymphocytic insulitis in pancreatic islets and pre-diabetic BB rats. Diabetologia 32: 126-134

33. Rothe H, Ongoren C, Martin S, Rosen P and Kolb H (1994) Abnormal TNF-alpha production in diabetes-prone BB rats: enhanced TNF-alpha expression and defective PGE2 feedback inhibition. Immunology 81: 407-413

34. Feldmann M, Brennan FM and Maini R (1998) Cytokines in autoimmune disorders. Int. Rev. Immunol. 17: 217-228
35. Pakala SV, Chivetta M, Kelly CB and Katz JD (1999) In autoimmune diabetes the transition from benign to pernicious insulitis requires an islet cell response to tumor necrosis factor alpha. J. Exp. Med. 189: 1053-1062

36. Moore KJ, Yeh K, Naito T and Kelley VR (1996) TNF-alpha enhances colonystimulating factor-1-induced macrophage accumulation in autoimmune renal disease. J. Immunol. 157: 427-432

37. van Rees EP, Voorbij HA and Dijkstra CD (1988) Neonatal development of lymphoid organs and specific immune responses in situ in diabetes-prone BB rats. Immunology 65: 465-472

38. Serreze DV and Leiter EH (1991) Development of diabetogenic T cells from $\mathrm{NOD/Lt} \mathrm{marrow} \mathrm{is} \mathrm{blocked} \mathrm{when} \mathrm{an} \mathrm{allo-} \mathrm{H}-2$ haplotype is expressed on cells of hemopoietic origin, but not on thymic epithelium. J. Immunol. 147: 1222-1229

39. Serreze DV, Gaedeke JW and Leiter EH (1993) Hematopoietic stem cell defects underlying abnormal macrophage development and maturation in NOD/Lt mice: defective regulation of cytokine receptors and protein kinase C. Proc. Natl. Acad. Sci. USA 90: $9625-9629$

40. Piganelli JD, Martin T and Haskins K (1998) Splenic macrophages from the NOD mouse are defective in the ability to present antigen. Diabetes 47: 1212-1218

41. Kerr JFR, Wyllie AH and Currie AR (1972) Apoptosis: A basic biological phenomenon with wide-ranging implications in tissue kinetics. Br. J. Cancer 26 : $239-257$

42. Meagher LC, Savill JS, Baker A, Fuller RW and Haslett C (1992) Phagocytosis of apoptotic neutrophils does not induce macrophage release of thromboxane B2. J. Leukoc. Biol. 52: 269-273

43. Fadok VA, Bratton DL, Konowal A, Freed PW, Westcott JY and Henson PM (1998) Macrophages that have ingested apoptotic cells in vitro inhibit proinflammatory cytokine production through autocrine/paracrine mechanisms involving TGF-beta, PGE-2 and PAF. J. Clin. Invest. 101: 890-898

44. Casciola-Rosen LA, Anhalt G and Rosen A (1994) Autoantigens targeted in systemic lupus erythematosus are clustered in two populations of surface structures on apoptotic keratinocytes. J. Exp. Med. 179: 1317-1330

45. Mevorach D, Zhou JL, Song X and Elkon KB (1998) Systemic exposure to irradiated apoptotic cells induces autoantibody production. J. Exp. Med. 188: 387-392

46. Albert ML, Pearce SFA, Franciso LM, Sauter B, Roy P, Silverstein RL and Bhardwaj N (1998) Immature dendritic cells phagocytose apoptotic cells via $\alpha \mathrm{v} \beta 5$ and CD36, and cross-present antigens to cytotoxic T lymphocytes. J. Exp. Med. 188: $1359-1368$ 